\title{
Routing Strategies for Wireless Sensor Networks
}

\author{
Raúl Aquino-Santos ${ }^{1}$, Luis Villasenor-Gonzalez ${ }^{2}$, Jaime Sanchez ${ }^{2}$, José Rosario \\ Gallardo ${ }^{2}$ \\ ${ }^{1}$ Faculty of Telematics, University of Colima \\ 28040, Av. Universidad 333, Colima, Colima, México \\ ${ }^{2}$ CICESE, Research Centre \\ 22860, Carretera Tijuana-Ensenada, k. 113 \\ Emsenada, B.C.N., Mexico \\ aquinor@ucol.mx; \{luisvi, jasan, jgallard@cicese.mx\}
}

\begin{abstract}
This paper evaluates three routing strategies for wireless sensor networks: source, shortest path, and hierarchical-geographical, which are the three most commonly employed by wireless ad-hoc and sensor networks algorithms. Source routing was selected because it does not require costly topology maintenance, while shortest path routing was chosen because of its simple discovery routing approach and hierarchical-geographical routing was elected because it uses location information via Global Positioning System (GPS). The performance of these three routing strategies is evaluated by simulation using OPNET, in terms of latency, End to End Delay (EED), packet delivery ratio, routing overhead, overhead and routing load.
\end{abstract}

Keywords: Wireless sensor networks, multi-hop networks, unicast routing, hierarchical and flat routing mechanisms.

\section{Introduction}

Recent advances in micro-electro-mechanical systems (MEMS) technology have made the deployment of wireless sensor nodes a reality [1] [2], in part, because they are small, inexpensive and energy efficient. Each node of a sensor network consists of three basic subsystems: a sensor subsystem to monitor local environment parameters, a processing subsystem to give computation support to the node, and a communication subsystem to provide wireless communications to exchange information with neighboring nodes. Because each individual sensor node can only cover a relatively limited area, it needs to be connected with other nodes in a coordinated manner to form a sensor network (SN), which can provide large amounts of detailed information about a given geographic area. Consequently, a wireless sensor network (WSN) can be described as a collection of intercommunicated wireless sensor nodes which coordinate to perform a specific action. Unlike traditional wireless networks, WSNs depend on dense deployment and coordination to carry out their task. Wireless sensor nodes measure conditions in the environment surrounding them and then transform these measurements into signals that can be 
processed to reveal specific information about phenomena located within a coverage area around these sensor nodes.

WSNs have a variety of applications. Examples include environmental monitoring which involves monitoring air, soil and water, condition-based maintenance, habitat monitoring (determining the plant and animal species population and behavior), seismic detection, military surveillance, inventory tracking, smart spaces, etc. [3][4]. Despite their many diverse applications, WSNs pose a number of unique technical challenges due to the following factors: fault tolerance (robustness), scalability, production costs, operating environment, sensor network topology, hardware constraint, transmission media and power consumption.

To date, the ZigBee Alliance is developing a communication standard for WSNs to support low-cost, low-power consumption, two-way wireless communications. Solutions adopting the ZigBee standard will be embedded in consumer electronics, home and building automation, industrial controls, PC peripherals, medical sensor applications, toys and games [5].

Sensor networks are generally deployed into an unplanned infrastructure where there is no a priori knowledge of their specific location. The resulting problem of estimating the spatial coordinates of the node is referred to as location. Most of the proposed localization methods today depend on recursive trilateration/multilateration techniques [6].

In WSNs, obtaining data is sometimes more important than knowing the specific Id of the originating node. Because the data collected by many sensors in WSNs is typically based on a common phenomenon, there is a high probability that this data has some degree of redundancy. Data redundancy needs to be exploited by the routing protocol to optimize energy and bandwidth utilization.

Many researchers are currently engaged in developing strategies to meet these many diverse requirements. This paper focuses on a performance analysis of three basic routing strategies which are commonly used in routing protocols in wireless ad-hoc and sensor networks. The remainder of the paper is organized as follows: Section 2 considers various routing protocols that deal with state-of-the-art routing techniques for wireless sensor networks. Section 3 provides an explanation of the scenario simulated and finally, Section 4 summarizes our work and proposes future research.

\section{State-of-the-art of Routing Techniques for Wireless Sensor Networks}

Routing protocols for wireless sensor networks can be classified as data-centric, hierarchical or location-based [7]. In these three categories, source, shortest path, and hierarchical-geographical strategies play an important role to develop all of the routing protocols. 


\subsection{Data-centric protocols}

In data-centric protocols, the sensor nodes broadcast an advertisement for the available data and wait for a request from an interested sink. Flooding is a simple technique that can be used to broadcast information in wireless sensor networks, however it requires significant resources because each node receiving a message must rebroadcast it, unless a maximum number of hops for the packet are reached, or the destination of the packet is the node itself. Flooding is a reactive technique that does not require costly topology maintenance or complex route discovery algorithms. However, it does have several additional deficiencies such as: implosion, overlap and resource blindness [8]. A derivation of flooding is gossiping, in which nodes do not broadcast. Instead, they send the incoming packets to a randomly selected neighbor.

Sensor protocols for information via negotiation (SPIN) address the deficiencies of classic flooding by providing negotiation and resource adaptation [9]. However, SPIN data advertisement mechanism cannot, by itself, guarantee data delivery [10]. SPIN employs a shortest path strategy based on three types of messages to communicate:

ADV - new data advertisement. When a SPIN node has data to share, it can advertise this fact by transmitting an ADV message containing meta-data.

REQ - request for data. A SPIN node sends an REQ message when it wishes to receive some actual data.

DATA - data message. DATA messages contain actual sensor data with a meta-data header.

Unlike traditional networks, a sensor node does not necessarily require an identity (e.g. an address). Instead, applications focus on the different data generated by the sensors. Because data is identified by its attributes, applications request data by matching certain attribute values. One of the most popular algorithms for data-centric protocols is direct diffusion and it bases its routing strategy on shortest path [11]. A sensor network based on direct diffusion exhibits the following properties: each sensor node names data that it generates with one or more attributes, other nodes may express interests based on these attributes, and network nodes propagate interests. Interests establish gradients that direct the diffusion of data. In its simple form, a gradient is a scalar quantity. Negative gradients inhibit the distribution of data along a particular path, and positive gradients encourage the transmission of data along the path.

The Energy-Aware Routing protocol is a destination-initiated reactive protocol that increases the network lifetime using only one path at all times, it seems very similar to source routing [12]. Rumor routing [13] is a variation of direct diffusion that is mainly intended for applications where geographic routing is not feasible. Gradientbased routing is another variant of direct diffusion [14]. The key idea of gradientbased routing is to memorize the number of hops when the interest is diffused throughout the network. Constraint Anisotropic Diffusion Routing (CADR) is a general form of direct diffusion [15] and lastly, Active Query Forwarding in Sensor Networks (ACQUIRE) [16] views the network as a distributed database, where complex queries can be further divided into several sub queries. 


\subsection{Hierarchical protocols}

Hierarchical protocols are based on clusters because clusters can contribute to more scalable behavior as the number of nodes increases, provide improved robustness, and facilitate more efficient resource utilization for many distributed sensor coordination tasks.

Low-Energy Adaptive Clustering Hierarchy (LEACH) is a cluster-based protocol that minimizes energy dissipation in sensor networks by randomly selecting sensor nodes as cluster-heads [17]. Power-Efficient Gathering in Sensor Information System (PEGASIS) [18] is a near optimal chain-based protocol. The basic idea of the protocol is to extend network lifetime by allowing nodes to communicate exclusively with their closest neighbors, employing a turn-taking strategy to communicate with the Base Station (BS). Threshold-sensitive Energy Efficient protocol (TEEN) [19] and Adaptive Periodic TEEN (APTEEN) [20] have also been proposed for time-critical applications. In TEEN, sensor nodes continuously sense the medium, but data transmission is done less frequently. APTEEN, on the other hand, is a hybrid protocol that changes the periodicity or threshold values used in the TEEN protocol, according to user needs and the application type.

\subsection{Location-based protocols}

In location-based routing, the forwarding decision by a node is primarily based on the position of a packet's destination and the position of the node's immediate onehop neighbor. The position of the destination is contained in the header of the packet. If a node has a more accurate position of the destination, it may choose to update the position in the packet before forwarding it. The position of the neighbors is typically learned through a one-hop broadcast beacon. These beacons are sent periodically by all nodes and contain the position of the sending node.

We can distinguish three main packet-forwarding strategies for position-based routing: greedy forwarding, restricted directional flooding, and hierarchical approaches. For the first two, a node forwards a given packet to one (greedy forwarding) or more (restricted directional flooding) one-hop neighbors that are located closer to the destination than the forwarding node itself. The selection of the neighbor in the greedy case depends on the optimization criteria of the algorithm. The third forwarding strategy is to form a hierarchy in order to scale to a large number of mobile nodes.

Minimum Energy Communication Network (MECN) [21] establishes and maintains a minimum energy network for wireless networks by utilizing low-power geographic positioning system (GPS). The main idea of MECN is to find the sub-network with the smallest number of nodes that requires the least transmission power between any two particular nodes (shortest path). The Small Minimum Energy Communication Network (SMECN) [22] is an extension of MECN. The major drawback with MECN is that it assumes every node can transmit to every other node, which is not always possible. One advantage of SMECN is that it considers obstacles between pairs of nodes. Geographic Adaptive Fidelity (GAF) [23] is an energy-aware location-based 
routing algorithm primarily designed for ad-hoc networks that can also be applied to sensor networks. GAF conserves energy by turning off unnecessary nodes in the network without affecting the level of routing fidelity. Finally, Geographic and Energy Aware Routing [24] uses energy-awareness and geographically informed neighbor selection heuristics to route a packet toward the destination region.

\subsection{ZigBee Protocol}

The IEEE 802.15.4-2003 standard defines the lower two layers: the physical (PHY) layer and the medium access control (MAC) sub-layer. The ZigBee alliance builds on this foundation by providing the network (NWK) layer and the framework for the application layer, which includes the application support sub-layer (APS), the ZigBee device objects $(\mathrm{ZDO})$ and the manufacturer-defined application objects.

IEEE 802.15.4-2003 has two PHY layers that operate in two separate frequency ranges: $868 / 915 \mathrm{MHz}$ and $2.4 \mathrm{GHz}$. The $2.4 \mathrm{GHz}$ mode specifies a Spread Spectrum modulation technique with processing gain equal to 32 . It handles a data rate of 250 kbps, with Offset-QPSK modulation, and a chip rate of 2 Mcps.

The $868 / 915 \mathrm{MHz}$ mode specifies a DSSS modulation technique with data rates of $20 / 40 \mathrm{kbps}$ and chip rates of $300 / 600 \mathrm{kcps}$. The digital modulation is BPSK and the processing gain is equal to 15 .

On the other hand, the MAC sub-layer controls access to the radio channel using a CSMA-CA mechanism. Its responsibilities may also include transmitting beacon frames, synchronizing transmissions and providing a reliable transmission mechanism.

The responsibilities of the ZigBee NWK layer includes mechanisms used to join and exit a network, in order to apply security to frames and to route frames to their intended destinations based on shortest path strategy. In addition, the discovery and maintenance of routes between devices transfer to the NWK layer. Also, the discovery of one-hop neighbors and the storing of pertinent neighbor information are done at the NWK layer. The NWK layer of a ZigBee coordinator is responsible for starting a new network, when appropriate, and assigning addresses to newly associated devices.

The responsibilities of the APS sub-layer include maintaining tables for binding, which is the ability to match two devices together based by their services and their needs, and forwarding messages between bound devices. The responsibilities of the ZDO include defining the role of the device within the network, initiating and/or responding to binding requests and establishing a secure relationship between network devices. The ZDO is also responsible for discovering devices on the network and determining which application services they provide. 


\section{Scenarios Simulated}

The routing protocols described in section 2 make use of one, or a combination of the following strategies: source, shortest path or hierarchical-geographical routing strategies. The performance of these basic strategies is evaluated using the following metrics:

- Route discovery time (Latency): is the time the sink has to wait before actually receiving the first data packet.

- Average end-to-end delay of data packets: are all possible delays caused by queuing, retransmission delays at the MAC and propagation and transfer times.

- Packet delivery ratio: is the ratio of the number of data packets delivered to the destination and the number of data packets sent by the transmitter. Data packets may be dropped en route for several reasons: e.g. the next hop link is broken when the data packet is ready to be transmitted or one or more collisions have occurred.

- Routing load: is measured in terms of routing packets transmitted per data packets transmitted. The latter includes only the data packets finally delivered at the destination and not the ones that are dropped. The transmission at each hop is counted once for both routing and data packets. This provides an idea of network bandwidth consumed by routing packets with respect to "useful" data packets.

- Routing overhead: is the total number of routing packets transmitted during the simulation. For packets sent over multiple hops, each packet transmission (hop) counts as one transmission.

- Overhead (packets): is the total number of routing packets generated divided by the sum of total number of data packets transmitted and the total number of routing packets

\subsection{Basic Routing Strategies implemented}

In source routing, each packet header carries the complete ordered list of nodes through which the packet must pass. The key advantage of source routing is that intermediate nodes do not need to maintain up-to-date routing information in order to route the packets they forward, since the packets themselves already contain all the routing information. This fact, coupled with the on-demand nature of the protocol, eliminates the need for the periodic route advertisement and neighbor detection packets present in other protocols such as the Energy Aware Routing.

In the shortest path strategy, when a node $S$ needs a route to destination $D$, it broadcasts a route request message to its neighbors, including the last known sequence number for that destination. The route request is flooded in a controlled manner through the network until it reaches a node that has a route to the destination. 
Each node that forwards the route request creates a reverse route for itself back to node S. Examples are SPIN, Direct Diffusion, MECN, and the ZigBee standard.

When the route request reaches a node with a route to $D$, that node generates a route reply containing the number of hops necessary to reach $D$ and the sequence number for D most recently seen by the node generating the reply. Importantly, each node that forwards this reply back toward the originator of the route request (node S) creates a forward route to $D$. The state created in each node remembers only the next hop and not the entire route, as would be done in source routing.

Hierarchical-geographical strategy improves the traditional routing strategies based on non-positional routing by making use of location information provided by GPS as it minimizes flooding of its Location Request (LREQ) packets. Flooding, therefore, is directive for traffic control by using only the selected nodes, called gateway nodes to diffuse LREQ messages. The purpose of gateway nodes is to minimize the flooding of broadcast messages in the network by reducing duplicate retransmissions in the same region.

Member nodes are converted into gateways when they receive messages from more than one cluster-head. All the members of the cluster read and process the packet, but do not retransmit the broadcast message. This technique significantly reduces the number of retransmissions in a flooding or broadcast procedure in dense networks. Therefore, only the gateway nodes retransmit packets between clusters (hierarchical organization). Moreover, gateways only retransmit a packet from one gateway to another in order to minimize unnecessary retransmissions, and only if the gateway belongs to a different cluster-head.

We decided to evaluate source, shortest path and hierarchical-geographical routing strategies since they represent the foundation of all of the above mentioned routing protocols.

The simulator for evaluating the three routing strategies for our wireless sensor network is implemented in OPNET 11.5, and the simulation models a network of 225 MICAz sensor nodes [2]. This configuration represents a typical scenario where nodes are uniformly placed within an area of $1.5 \mathrm{~km} 2$.

We used a $2405-2480 \mathrm{MHz}$ frequency range and a $250 \mathrm{kbps}$ data rate for our simulation, with a MICAz sensor node separation of $75 \mathrm{~m}$. This scenario represents a typical wireless sensor network with one sink node acting as a gateway to communicate the WSN with a separate network (Internet). In our scenario one sensor node communicates with the sink, and the sensor node sends a packet every second (constant bit rate).

\subsection{Simulation Results}

Figure 1 shows the latency between the sink and the source in milliseconds. Source and shortest path routing strategies show a similar behavior. However, hierarchicalgeographical routing shows the poorest behavior due to the transmission of position information via hello packets which produce more collision in the wireless medium, in addition, the cluster formation mechanism also increase the latency. 


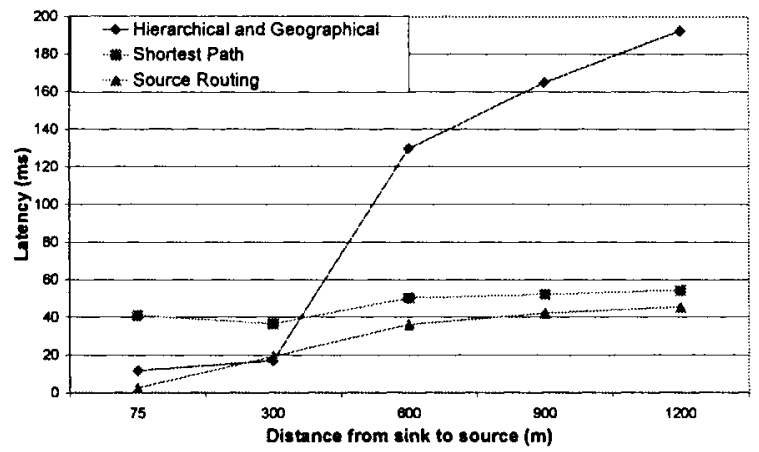

Fig. 1. Latency (milliseconds).

Figure 2 shows the End-to-End Delay (EED) between the sink and the source in milliseconds. The hierarchical-geographical routing strategy shows the worst behavior because the static nature of the wireless sensor nodes causes synchronization of the packets. Synchronization arises from the simultaneous transmission of packets between neighbors. As results, the frequent transmission of Hello packets produces more collision with data packets.

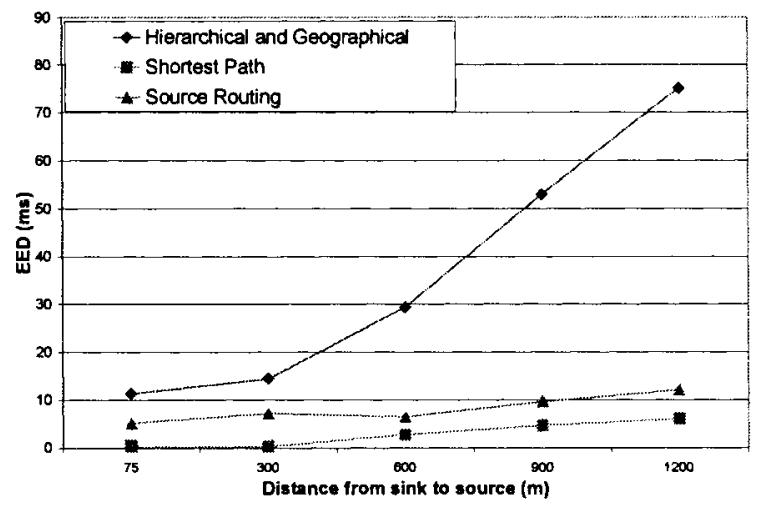

Fig. 2. End-to-End Delay (milliseconds)

The three routing mechanisms show a similar behavior in terms of packet delivery ratio because of their static nature, as illustrated in figure 3 . 


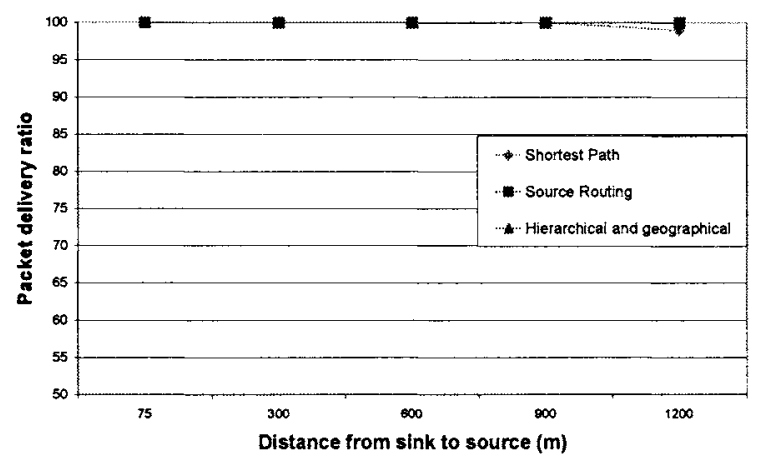

Fig. 3. Packet delivery ratio.

Figure 4 shows the Routing Overhead between the sink and the source. Routing overhead is the total number of routing packets transmitted during the simulation. Again, the shortest path routing strategy performs the best and the hierarchicalgeographical strategy the worst. This is due to the Hello packets used for the cluster formation mechanism.

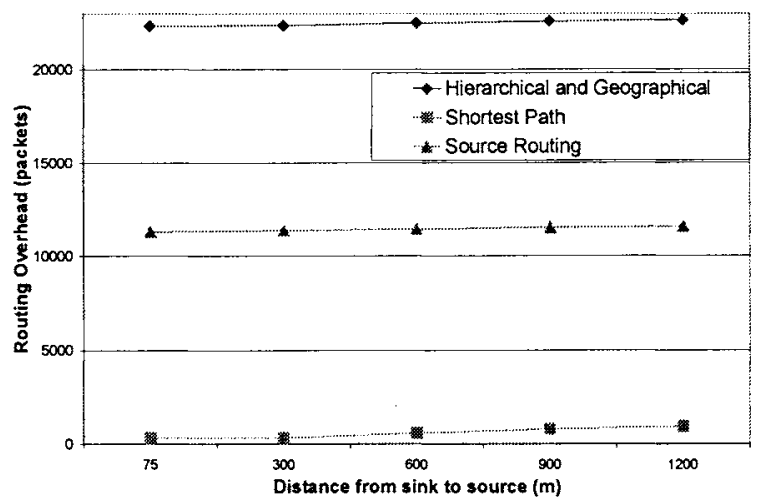

Fig. 4. Routing Overhead (packets).

Figure 5 shows the overhead between the sink and the source. The shortest path technique also has the best performance, with source routing and hierarchicalgeographical mechanism performing in a similar fashion. 


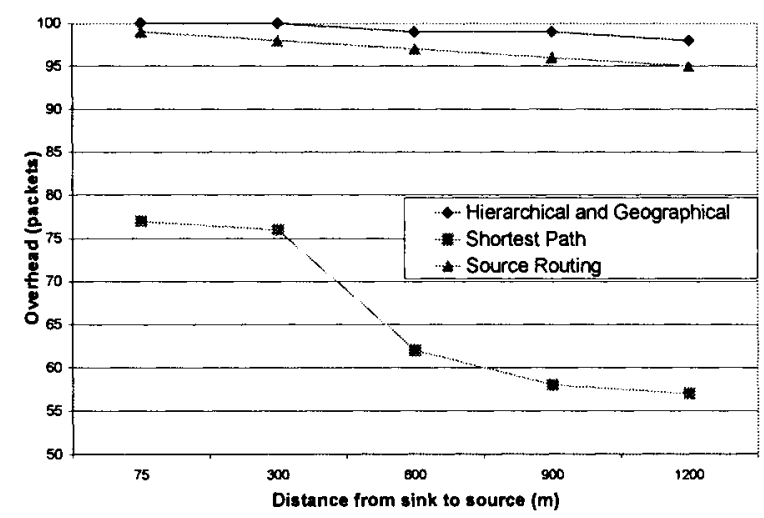

Fig. 5. Overhead (packets).

Figure 6 shows the Routing Load between the sink and the source. This metric provides an idea of how much network bandwidth is consumed by routing packets in relation to the useful data packets actually received. Once again, the shortest path routing strategy has the best performance, and the hierarchical-geographical mechanism the worst.

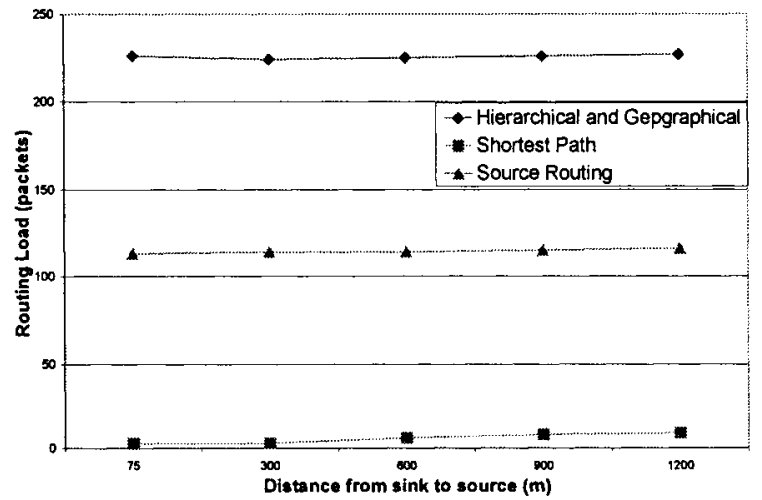

Fig. 6. Routing Load (packets).

\section{Conclusions and Future Work}

In this paper, we have evaluated three basic routing strategies widely used in routing protocols for wireless sensor networks. Source routing only improves shortest path and hierarchical-geographical routing in terms of latency. The main disadvantage of 
source routing is that it lacks a number of hops metric, which can frequently result in longer path selection. Shortest path behaves well in terms of EED, routing overhead, overhead and routing load. Hierarchical-geographical routing performs the worst because it must send hello packets in order to acquire and transmit location information. This consideration makes hierarchical-geographical routing in wireless sensor networks more weighty because it transmits hello packets more frequently, requiring greater bandwidth and energy resources. However, despite these significant disadvantages, hierarchical-geographical routing remains the routing option most often used in health, military, agriculture, robotic, environmental and structural monitoring. An important area of future research is to optimize hierarchicalgeographical routing algorithm to facilitate its use in large geographical areas requiring dense sensor distribution.

\section{Acknowledgements}

The authors acknowledge the support received by the National Council of Science and Technology (CONACYT) under project grant No. 48391.

\section{References}

1. V. Rajaravivarma, Yi Yang, and Teng Yang. An Overview of Wireless Senor Network and Applications. Proceeding of the 35th Southeastern Symposium on System Theory, pp. 432436, 2003.

2. http://www.xbow.com/Products/Wireless_Sensor_Networks.htm

3. Stephan Olariu and Qingwen Xu. Information Assurance in Wireless Sensor Networks. Proceedings of the 19th IEEE International Parallel and Distributed Processing Symposium, pp. $236-240,2005$.

4. Alan Mainwaring, Joseph Polastre, Robert Szewczyk, David Culler, John Anderson. Wireless Sensor Networks for Habitat Monitoring. Proceeding of the 1st ACM International workshop on wireless sensor Networks and applications, pp. 88-97, 2002.

5. ZIgBee Specification. ZigBee Document 053474r06, version 1.0. December 2004.

6. Nirupama Bulusu, John Heidemann, Deborah Strin. GPS-less Low Cost Outdoor Localization For Very Small Devices. IEEE Personal Communication, vol. 7, issue 5, pp. 28-34, 2000.

7. A Reliable Routing Protocol Design for wireless sensor Networks. Yanjun Li, Jiming Chen, Ruizhong Lin, Zhi Wang. International Conference on Mobile Ad-hoc and Sensor Systems, 2005.

8. I.F. Akyildiz, W. Su, Y. Sankarasubramaniam, E. Cayirci. Wireless sensor networks: a survey. Computer Networks, pp. 393-422, 2002.

9. Wendi Rabiner Heinzelman, Joana Kulik, and Hari Balakrishnan. Adaptive Protocols for Information Dissemination in Wireless Sensor Networks. Proceedings of the 5th annual ACM/IEEE International Conference on Mobil Computing and Networking (MOBICOM), pp. 174-185, 1999.

10.Jamal N. Al-karaki, Ahmed E. Kamal. Routing Techniques in Wireless Sensor Networks: A survey. IEEE Wireless Communications, pp. 6-28, 2004. 
11.Deborah Estrin, Ramesh Govindan, John Heidemann, Satish Kumar. Next Century Challenges: Scalable Coordination in Sensor Networks. Proceedings of the 5th ACM/IEEE International Conference on Mobile Computing and Networking, pp. 263-270, 1999.

12.Shah, R. C. Rabaey, J. M. Energy Aware Routing for low Ad Hoc Sensor Networks. IEEE Wireless Comunications and Networks Conference, vol. 1, pp. 350-355, 2002.

13.David Braginsky, Deborah Estrin. Rumor Routing Algorithm for Sensor Netorks. International Conference on Distributed Computing Systems (ICDCS-22), 2002.

14.Curt Schurgers, Mani B. Srivastava. Energy Efficient Routing in Wireless Sensor Networks. Proceeding of the Communication for Network-centric operations: creating the information force, 2001.

15.Maurice Chu, Horst Haussecker, and Feng Zhao. Scalable Information-Driven Sensor Querying and Routing for ad hoc Heterogeneous Sensor Networks. International Journal of High Performance Computing Applications, 2002.

16.Narayanan Sadagopan, Bhaskar Krishnamachari, and Ahmed Helmy. The ACQUIRE Mechanism for Efficient Querying in Sensor Networks. Proceedings of the IEEE Intemational Workshop on Sensor Network Protocols and Applications (SNPA), in conjunction with IEEE ICC, pp. 149-155, 2003.

17. Heinzelman, W. R. Chandrakasan, A. Balakrishnan, H. Energy-efficient communication protocol for wireless microsensor networks. Proceedings of the 33rd Annual Hawaii International Conference on System Sciences, vol. 2, pp. 1-10, 2000.

18.Stephanie Lindsey and Cauligi S. Raghavendra. PEGASIS: Power-Efficient GAthering in Sensor Information Systems. Proceeding of the IEEE Aerospace Conference, vol. 3, pp. 1125-1130, 2002.

19.Arati Manjeshwar and Dharma P. Agrawal. TEEN: A Routing Protocol for Enhanced Efficiency in Wireless Sensor Networks. Proceedings of the 15th International Symposium on Parallel and Distributed Processing, pp. 2009-2015, 2001.

20.Arati Manjeshwar and Dharma P. Agrawal. APTEEN: A Hybrid Protocol for Efficient Routing and Comprehensive Information Retrieval in Wireless Sensor Networks, Proceedings of the 16th International Symposium on Parallel and Distributed Processing, pp.195-202, 2002.

21.Volkan Rodoplu and Teresa H. Meng. Minimum Energy Mobile Wireless Networks. IEEE Journal on selected areas in communications, vol. 17, issue 8, pp. 1333-1344, 1999.

22.Li Li, Joseph Y. Halpern. Minimum-Energy Mobile Wireless Networks Revisited. IEEE International Conference on Communications, vol. 1, pp. 278-283, 2001.

23. Ya Xu, John Heideman, and Deborah Estrin. Geography-informed Energy Conservation for Ad-Hoc Routing. In proceedings of the ACM/IEEE International Conference on Mobile Computing and Networking, pp. 70-84, 2001.

24.Yan Yu, Ramesh Govindan, Deborah Estrin. Geographic and Energy Aware Routing: a recursive data dissemination protocol for wireless sensor networks. UCLA Computer Science Department Technical Report UCLA/CSD-TR-01-0023, 2001. 